Supporting Information

\title{
Designing Porous Materials to Resist Compression: Mechanical Reinforcement of a Zr-MOF with Structural Linkers
}

Lee Robison ${ }^{1,2}$, Riki J. Drout ${ }^{1}$, Louis R. Redfern ${ }^{1}$, Florencia A. Son ${ }^{1}$, Megan C. Wasson ${ }^{1}$, Subhadip Goswami $^{1}$, Zhijie Chen ${ }^{1}$, Alyssa Olszewski ${ }^{1}$, Karam Idrees ${ }^{1}$, Timur Islamoglu ${ }^{1}$, and Omar K. Farha $^{1,3 *}$

${ }^{1}$ Department of Chemistry and International Institute for Nanotechnology, Northwestern University, 2145 Sheridan Road, Evanston, Illinois 60208, United States

${ }^{2}$ X-ray Science Division, Advanced Photon Source, Argonne National Laboratory, Argonne, Illinois 60439-4858, United States

${ }^{3}$ Department of Chemical and Biological Engineering, Northwestern University, 2145 Sheridan Road, Evanston, IL 60208, USA

*Corresponding Author's Email: o-farha@,northwestern.edu 


\section{Table of Contents}

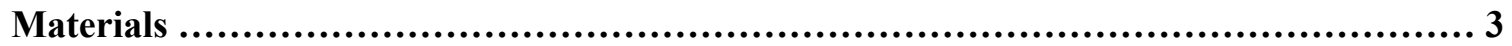

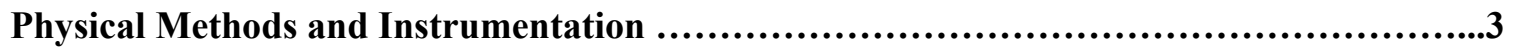

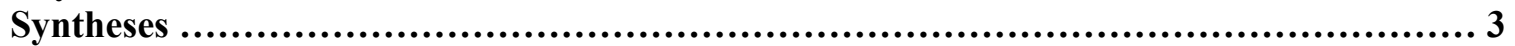

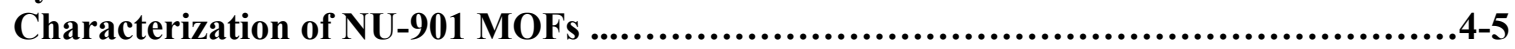

Solvent Assisted Linker Incorporation . ........................................................6

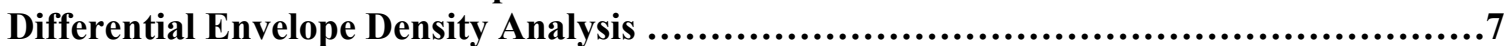

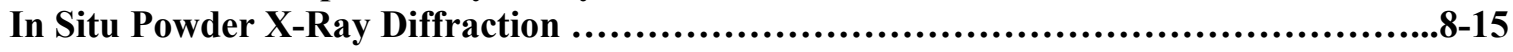

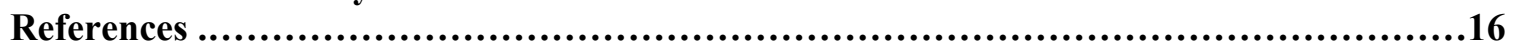




\begin{abstract}
Materials
Zirconium (IV) acetylacetonate, 4-aminobenzoic acid, 2,6-naphthalenedicarboxylic acid, Fluorinert ${ }^{\mathrm{TM}}$ FC70 (perfluorotripentylamine), and hydrochloric acid were purchased from Sigma Aldrich Chemicals Company, Inc. (Milwaukee, WI) and were used as received. The ligand for NU-901, 1,3,6,8-tetrakis(pbenzoic acid)pyrene ( $\left.\mathrm{H}_{4} \mathrm{TBAPy}\right)$, was synthesized by a published procedure ${ }^{1}$ and its purity was confirmed by ${ }^{1} \mathrm{H}-\mathrm{NMR}$ spectroscopy. Concentrated sulfuric acid was purchased from VWR Scientific, LLC (Chicago, IL). Acetone and N,N dimethylformamide (DMF) were obtained from Fisher Scientific and used without further purification. All gases used for the adsorption and desorption measurements were Ultra High Purity Grade 5, and all other gases, were obtained from Airgas Specialty Gases (Chicago, IL).
\end{abstract}

\title{
Physical Methods and Instrumentation
}

Ambient pressure powder X-ray Diffraction (PXRD) data were collected at the IMSERC X-ray Facility at Northwestern University on a STOE-STADI-MP powder diffractometer equipped with an asymmetric curved Germanium monochromator (CuK $\alpha 1$ radiation, $\lambda=1.54056 \AA$ ) and one-dimensional silicon strip detector (MYTHEN2 $1 \mathrm{~K}$ from DECTRIS). The line focused $\mathrm{Cu}$ X-ray tube was operated at $40 \mathrm{kV}$ and 40 $\mathrm{mA}$. Powder was packed in a $3 \mathrm{~mm}$ metallic mask and sandwiched between two layers of polyimide tape. Intensity data from 2 to 37 degrees $2 \theta$ were collected over a period of 10 mins. The instrument was calibrated against a NIST Silicon standard (640d) prior the measurement.

$\mathrm{N}_{2}$ adsorption and desorption isotherms were measured on a Micromeritics Tristar II 3020 (Micromeritics, Norcross, GA) instrument at $77 \mathrm{~K}$. Pore-size distributions were obtained using DFT calculations using a carbon slit-pore model with a $\mathrm{N}_{2}$ kernel in Micromeritics MicroActive software. Before each run, samples were activated at $120{ }^{\circ} \mathrm{C}$ for $16-24 \mathrm{~h}$ under high vacuum on a Smart Vacprep from Micromeritics. Approximately $40 \mathrm{mg}$ of sample was used in each measurement and Brunauer-Emmett-Teller (BET) surface area was calculated in the region $\mathrm{P} / \mathrm{P}_{0}=0.005-0.05$.

NMR samples were prepared by digesting $\sim 1-2 \mathrm{mg}$ with 5 drops of $\mathrm{D}_{2} \mathrm{SO}_{4}$ and diluting with $2 \mathrm{~mL}$ DMSO-d for ${ }^{1} \mathrm{H}-\mathrm{NMR}$ spectra.

Scanning electron microscopy (SEM) images were collected on a Hitachi SU8030 FE-SEM microscope with a field emission gun (Dallas, TX) at Northwestern University's EPIC/NUANCE facility. Before imaging, samples were coated with $\mathrm{OsO}_{4}$ to $\sim 9 \mathrm{~nm}$ thickness in a Denton Desk III TSC Sputter Coater (Moorestown, NJ).

\section{Syntheses}

NU-901 was prepared using a previous procedure with slight modifications. ${ }^{2} \mathrm{Zr}(\mathrm{acac})_{4}$ (acac $=$ acetyl acetonate) (146 mg, $0.3 \mathrm{mmol})$ and benzoic acid $(2.7 \mathrm{~g}, 22 \mathrm{mmol})$ were mixed in $8 \mathrm{~mL}$ of DMF in an 8dram vial and ultrasonically dissolved. The clear solution was incubated in an oven at $80{ }^{\circ} \mathrm{C}$ for $1 \mathrm{~h}$. After cooling down to room temperature H4TBAPy $(40 \mathrm{mg}, 0.06 \mathrm{mmol})$ was added and sonicated for $10 \mathrm{~min}$. The yellow suspension was placed in a pre-heated oven at $100{ }^{\circ} \mathrm{C}$ for $18 \mathrm{~h}$. After cooling down to room temperature, yellow polycrystalline material was isolated by centrifugation $(5 \mathrm{~min}, 7500 \mathrm{rpm})$ and solvent exchanged with fresh DMF three times $(10 \mathrm{~mL}$ each) followed by acetone three times $(10 \mathrm{~mL})$. Yellow polycrystalline NU-901 was collected by centrifugation and dried in a vacuum oven at $80{ }^{\circ} \mathrm{C}$ for $1 \mathrm{~h}$ (yield: $\sim 58 \mathrm{mg})$. 


\section{Characterization of NU-901 MOFs}

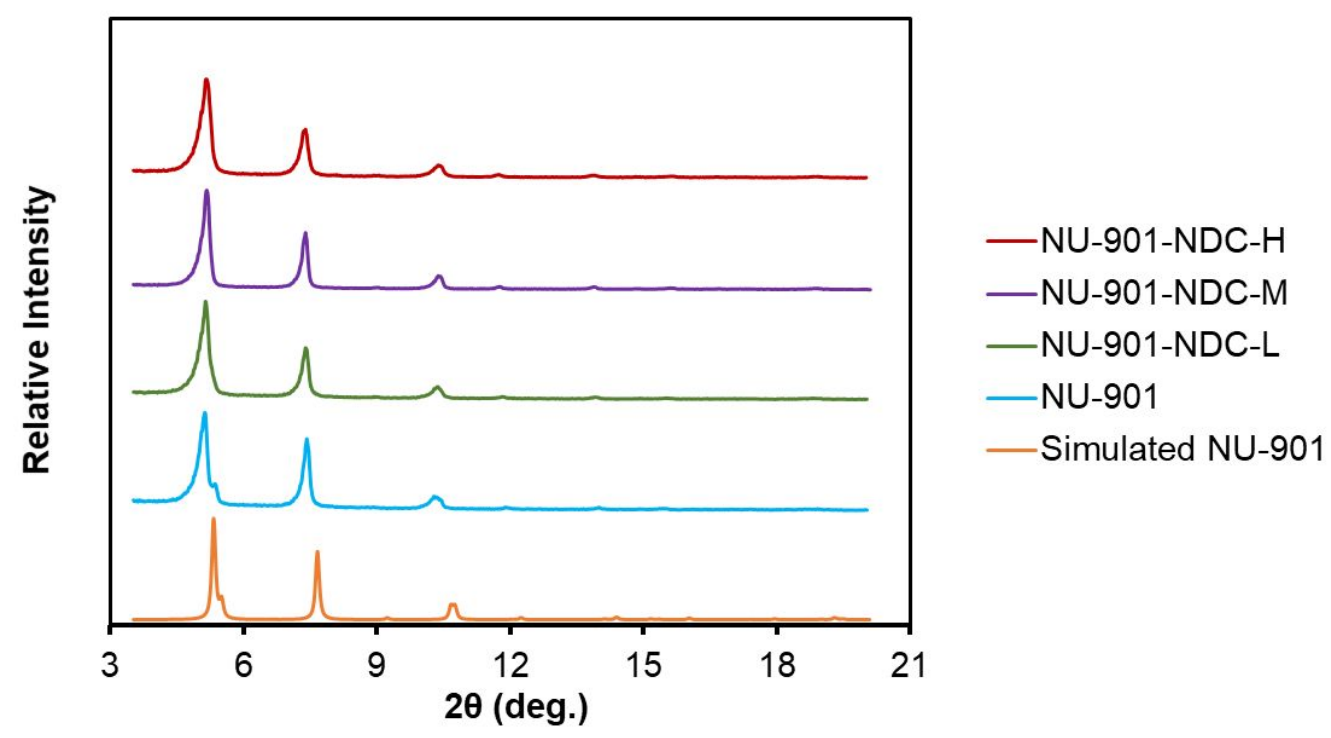

Figure S1. Ambient pressure powder X-Ray diffraction patterns for each NU-901 MOF.

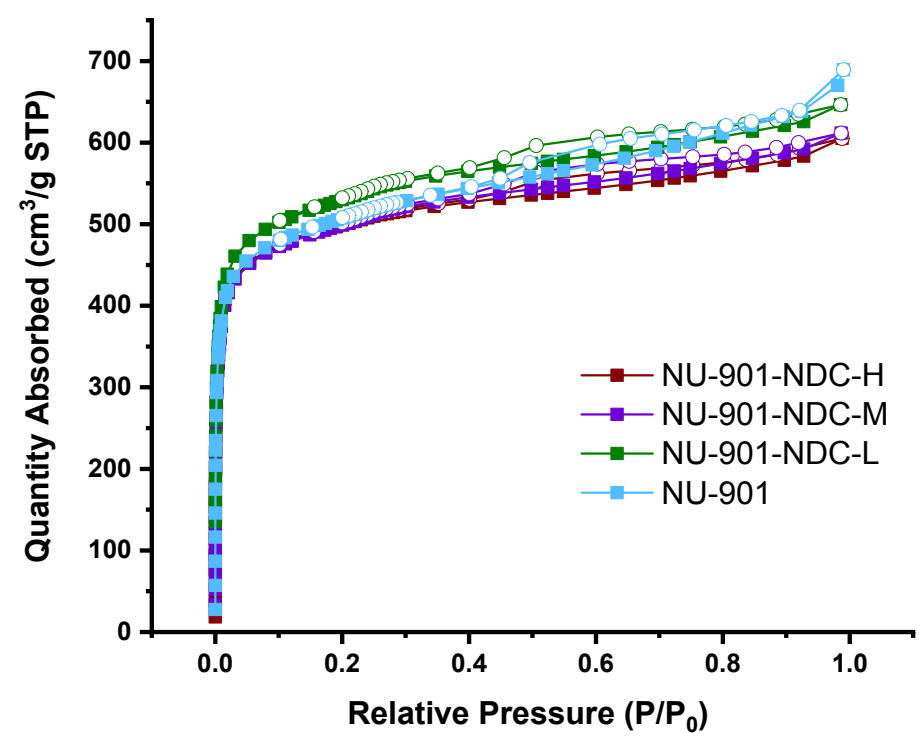

Figure S2. $\mathrm{N}_{2}$ isotherms for the NU-901 series. Closed circles denote adsorption data and open circles denote desorption data. 


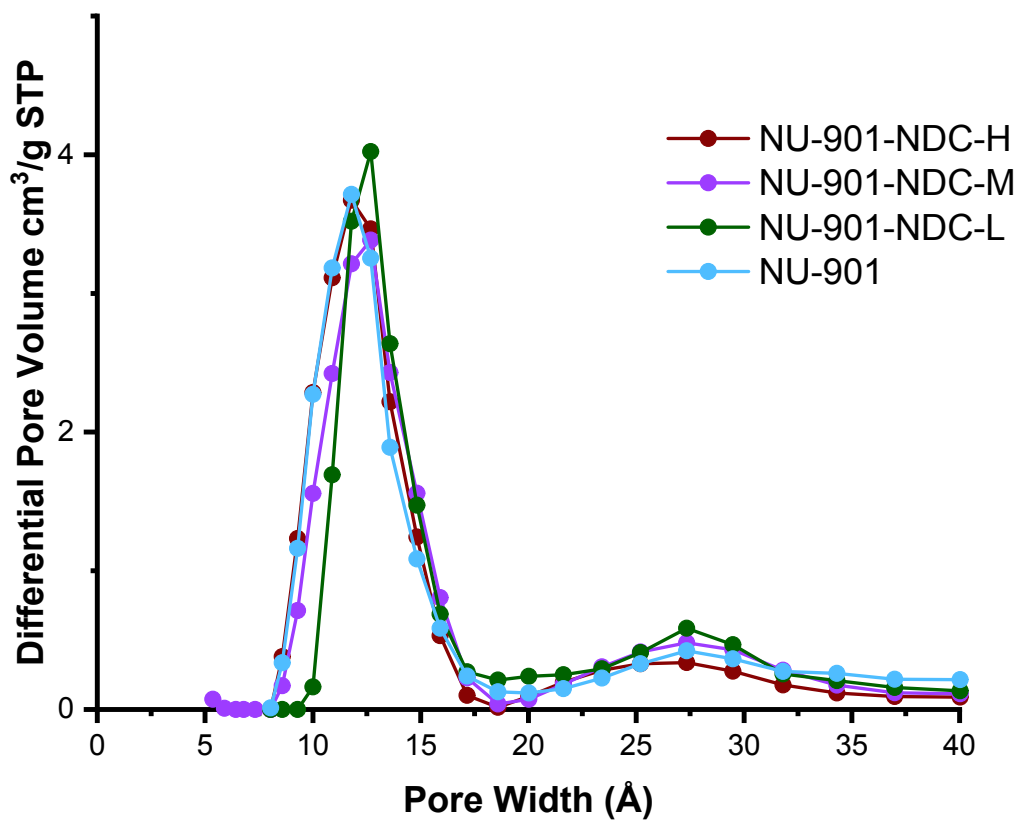

Figure S3. DFT pore size distributions for the NU-901 series.
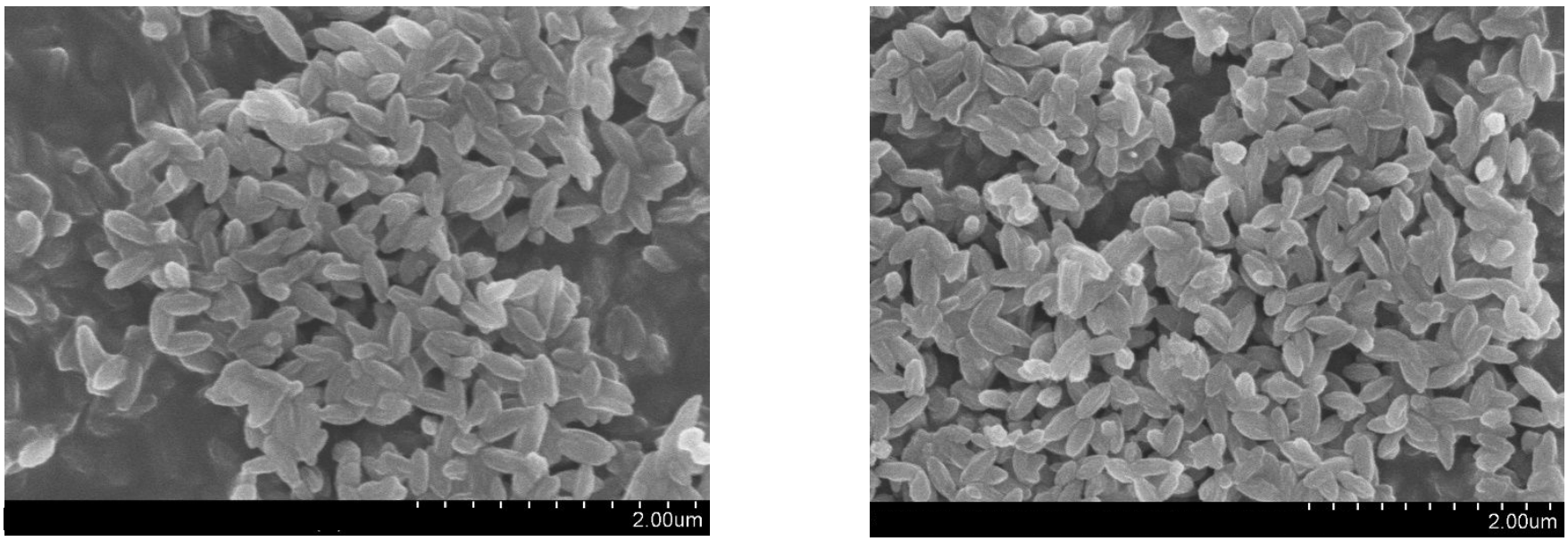

Figure S4. Scanning Electron Microscope Images of as synthesized NU-901. 


\section{Solvent Assisted Linker Incorporation}

Table S1. Incorporation of NDC ligands into NU-901 with various loading conditions determined by ${ }^{1} \mathrm{H}-\mathrm{NMR}$ spectroscopy. All samples were submerged in the appropriate amount of DMF and heated for $16 \mathrm{~h}$ at $80^{\circ} \mathrm{C}$.

\begin{tabular}{|c|cc|}
\hline Sample Name & $\begin{array}{c}\text { Exposure } \\
\text { (per } \mathbf{Z r}_{6} \text { Node) }\end{array}$ & $\begin{array}{c}\text { Incorporation Rate } \\
\text { (per } \mathbf{Z r}_{\mathbf{6}} \text { Node) }\end{array}$ \\
\hline NU-901 & $0 \mathrm{NDC}$ & $0 \mathrm{NDC}$ \\
NU-901-NDC-Low & $0.5 \mathrm{NDC}$ & $0.4 \mathrm{NDC}$ \\
NU-901-NDC-Medium & $1 \mathrm{NDC}$ & $0.7 \mathrm{NDC}$ \\
& & $1.1 \mathrm{NDC}$ \\
\hline
\end{tabular}

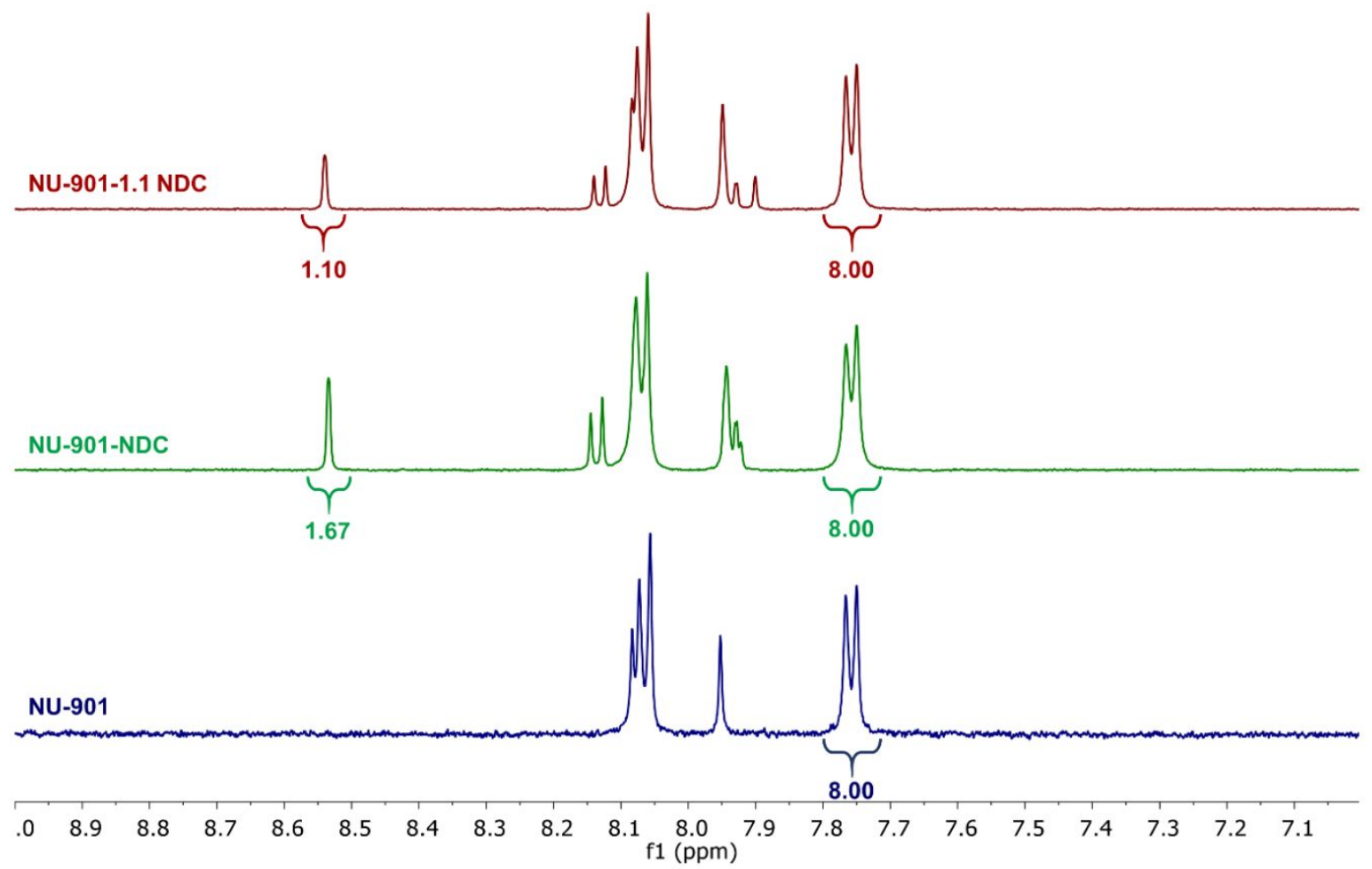

Figure S5. Representative ${ }^{1} \mathrm{H}-\mathrm{NMR}$ spectra of NU-901 (blue), NDC-SALI (green), and NDC-SALI$\mathrm{HCl}(\mathrm{red})$ after digestion in $\mathrm{a}_{2} \mathrm{SO}_{4} / \mathrm{DMSO}$ solution. Peaks used for quantification of NDC ligand are shown. 


\section{Differential Envelope Density Analysis}

Synchrotron powder X-ray diffraction (PXRD) data were collected at beamline 17-BM-B at the Advanced Photon Source (APS) at Argonne National Laboratory (ANL). The incident X-ray wavelength was 0.45418 $\AA$ A. Data were collected using a Perkin Elmer flat panel area detector (XRD 1621 CN3/EHS) over the angular range $0.5-15^{\circ} 2 \theta$. The measurements were taken at ambient temperature and pressure inside of a diamond

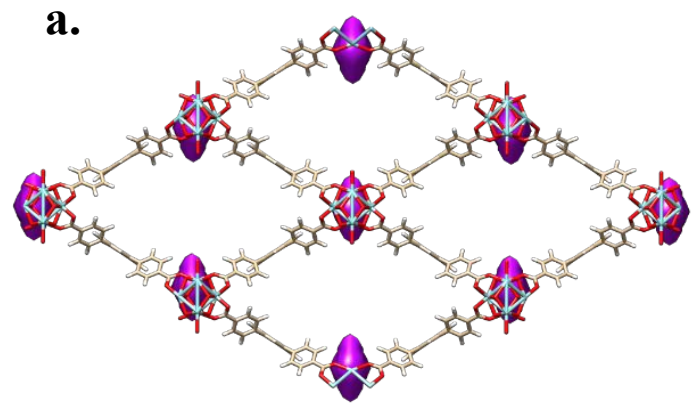

down $c$-axis

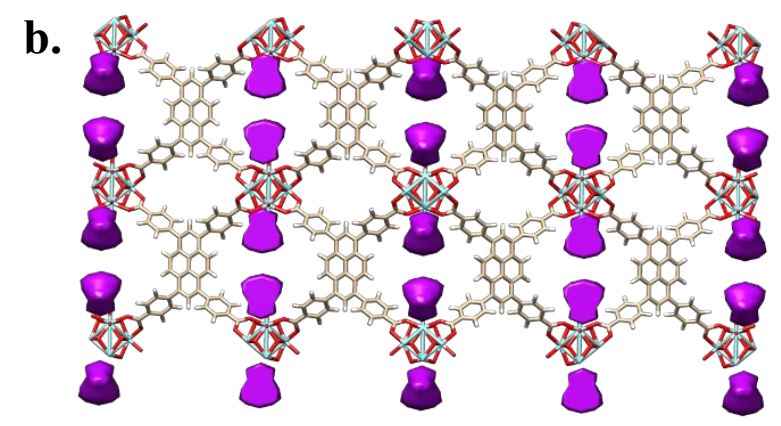

down $a$-axis

Figure S6. DED maps of (a) NU-901-NDC-H down the a-axis, and (b) NU-901-NDC-H down the $c$-axis. Electron density taken from the subtraction of NU-901 from NU-901-NDC is shown in purple.

anvil cell. Lattice parameters and Bragg scattering intensities of reflections out to $2 \theta=10^{\circ}$ were extracted via Le Bail whole pattern fitting of the NU-901 diffraction data. Structure envelopes (SEs) encompassing regions of high electron density were generated from extracted peak intensities, as previously described. ${ }^{3,4}$ To isolate contributions from the NDC added during SALI, a difference envelope density map (DEDs) was calculated via subtraction of the SE of the parent NU-901 material. The new electron density observed within the parent framework (purple) is observed only between the Zr-nodes along the $c$-axis. No additional electron density is found in the diamond pore of the MOF. The apparent gap in electron density of the NDC ligand in Figure $6 \mathrm{~b}$ within the c-pore can be rationalized by remembering that the largest contribution of electron density for the NDC ligand primarily resides on the carboxylic acid functional groups on either end of the structure, nearest the metal nodes, while the carbons in the center of the molecule are more electron poor and do not provide enough electron density to be subtracted from the parent structure with confidence. 


\section{In Situ Powder X-Ray Diffraction}

Each NU-901 MOF sample was mixed with $\mathrm{CaF}_{2}(\sim 10 \%$ by volume), which was used as an internal standard. The MOF and $\mathrm{CaF}_{2}$ powders were gently ground together in a mortar and pestle to ensure even distribution of the $\mathrm{CaF}_{2}$ standard and to break up any larger crystals or clumps of the respective samples. The mixture was then loaded into a $250 \mu \mathrm{m}$ hole in a $250 \mu \mathrm{m}$ thick stainless-steel gasket pre-indented to $100 \mu \mathrm{m}$ thickness using a membrane-driven diamond anvil cell (DAC) equipped with $500 \mu \mathrm{m}$ culet anvils. The gaskets were drilled using a laser micro-machining system at the High Pressure Collaborative Access Team (HP-CAT) at the Advanced Photon Source. ${ }^{5}$ The cell was closed, and the diffraction pattern was taken at ambient conditions. The cell was then opened, and a drop of Fluorinert ${ }^{\mathrm{TM}}$ FC-70 was added as a non-penetrating pressure transmitting fluid (hydrostatic limit: $1.0 \mathrm{GPa}$ ). The cell was sealed and in situ powder X-ray diffraction data were collected using the monochromatic X-rays $(\lambda=0.45418 \AA, 100 \mu \mathrm{m}$ beam size) at the 17-BM-B beamline at the Advanced Photon Source, Argonne National Laboratory in combination with a Perkin Elmer a-Si Flat Panel PE1621 area detector. Data were collected with 6 second exposures (1 min per image) as the pressure was varied from 0-1.0 GPa. After completion of a pressure campaign, the pressure within the cell was released and a final measurement was made at ambient pressure. Raw images were processed using GSAS-II, with sample-to-detector distance and tilt parameters based on the data obtained for $\mathrm{L} \mathrm{LaB}_{6}$ standard. ${ }^{6}$ The pressure-dependent lattice parameters were extracted from Le Bail fits of reported structural models to the diffraction data using GSAS-II. ${ }^{7}$ The parameters from each MOF sample are presented in Tables S2-S7, respectively. Equations of state were fit to the $\mathrm{P} v \mathrm{vs} . \mathrm{V}_{0} / \mathrm{V}_{\text {data }}$ using EOS-FIT7c and EOS-FIT7-GUI. ${ }^{8}, 9$ The 2nd-order Birch-Murnaghan equation of state provided the best fit for the data. While in situ diffraction data were collected to pressures up to $\sim 1.2 \mathrm{GPa}$, only the lowpressure regime presented could be effectively fit by a Birch-Murnaghan equation of state.

Table S2. Parameters of Le Bail fits to variable pressure powder diffraction data for NU-901

\begin{tabular}{|l|l|l|l|l|l|l|}
\hline Pressure (Gpa) & $\mathbf{V ~ C a F}_{\mathbf{2}}\left(\mathbf{\AA}^{3}\right)$ & $\mathbf{a ~ N U - 9 0 1}\left(\mathbf{\AA}^{3}\right)$ & $\mathbf{b ~ N U - 9 0 1}\left(\mathbf{\AA}^{3}\right)$ & $\mathbf{c ~ N U - 9 0 1}\left(\mathbf{\AA}^{3}\right)$ & $\mathbf{V} \mathbf{N U - 9 0 1}\left(\mathbf{\AA}^{3}\right)$ & $\mathbf{R w p}(\mathbf{\%})$ \\
\hline 0.000 & $163.235(7)$ & $34.18(2)$ & $19.89(1)$ & $16.580(5)$ & $11273(5)$ & 3.25 \\
$0.025(5)$ & $163.179(8)$ & $34.14(2)$ & $19.88(1)$ & $16.576(6)$ & $11249(5)$ & 3.41 \\
$0.062(5)$ & $163.108(8)$ & $34.07(2)$ & $19.86(1)$ & $16.566(6)$ & $11209(6)$ & 3.40 \\
$0.144(5)$ & $162.949(8)$ & $33.90(2)$ & $19.81(1)$ & $16.561(7)$ & $11125(7)$ & 3.66 \\
$0.205(5)$ & $162.832(8)$ & $33.72(2)$ & $19.760(9)$ & $16.538(7)$ & $11019(7)$ & 3.58 \\
$0.268(5)$ & $162.710(7)$ & $33.60(2)$ & $19.72(1)$ & $16.524(9)$ & $10947(8)$ & 4.01 \\
$0.343(5)$ & $162.564(7)$ & $33.49(2)$ & $19.660(9)$ & $16.512(9)$ & $10871(8)$ & 3.78 \\
$0.367(5)$ & $162.519(7)$ & $33.39(2)$ & $19.61(1)$ & $16.50(1)$ & $10801(8)$ & 3.86 \\
$0.428(6)$ & $162.401(9)$ & $33.34(2)$ & $19.56(1)$ & $16.47(1)$ & $10738(9)$ & 4.10 \\
$0.528(6)$ & $162.209(8)$ & $33.23(2)$ & $19.52(1)$ & $16.44(1)$ & $10660(9)$ & 3.98 \\
$0.594(6)$ & $162.083(9)$ & $33.14(2)$ & $19.47(1)$ & $16.42(1)$ & $10592(9)$ & 3.87 \\
\hline
\end{tabular}




\section{NU-901}

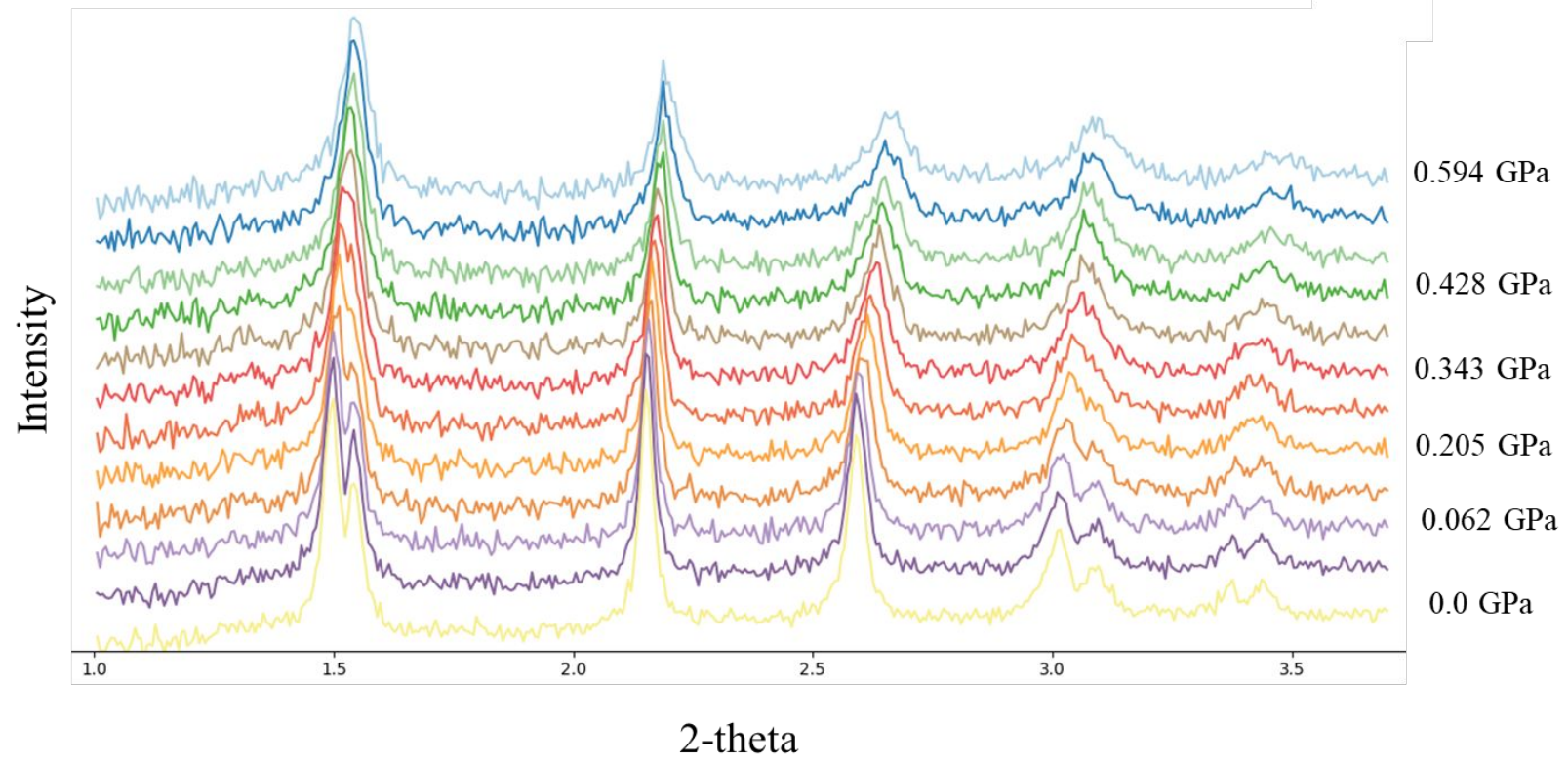

Figure S7. Powder diffraction data for NU-901 as pressure increases from 0.0-0.594 GPa.

Table S3. Parameters of Le Bail fits to variable pressure powder diffraction data for NU-901-NDCLow

\begin{tabular}{|c|c|c|c|c|c|c|}
\hline Pressure (Gpa) & $\mathrm{V} \mathrm{CaF}_{2}\left(\AA^{3}\right)$ & a NU-901 $\left(\AA^{3}\right)$ & b NU-901 $\left(\AA^{3}\right)$ & c NU-901 $\left(\AA^{3}\right)$ & V NU-901 $\left(\AA^{3}\right)$ & Rwp (\%) \\
\hline 0.000 & $163.005(8)$ & $34.39(2)$ & $19.34(2)$ & $16.77(2)$ & $11156(8)$ & 4.75 \\
\hline $0.037(5)$ & $162.925(7)$ & $34.30(2)$ & $19.32(2)$ & $16.78(2)$ & $11120(8)$ & 4.75 \\
\hline $0.090(6)$ & $162.822(9)$ & $34.16(2)$ & $19.33(2)$ & $16.76(2)$ & $11068(8)$ & 4.76 \\
\hline $0.141(7)$ & $162.72(1)$ & $34.09(2)$ & $19.33(2)$ & $16.74(2)$ & $11030(8)$ & 4.61 \\
\hline $0.163(7)$ & $162.68(1)$ & $34.07(3)$ & $19.34(2)$ & $16.72(2)$ & $11018(8)$ & 4.93 \\
\hline $0.173(7)$ & $162.66(1)$ & $34.09(3)$ & $19.31(2)$ & $16.72(2)$ & $11008(8)$ & 4.97 \\
\hline $0.185(6)$ & $162.639(9)$ & $34.04(3)$ & $19.33(2)$ & $16.71(2)$ & 10996(8) & 4.81 \\
\hline $0.190(6)$ & $162.629(9)$ & $34.04(3)$ & $19.33(2)$ & $16.70(2)$ & 10989(8) & 4.91 \\
\hline $0.197(6)$ & $162.617(8)$ & $34.05(3)$ & $19.32(2)$ & $16.71(2)$ & $10990(8)$ & 4.90 \\
\hline $0.205(6)$ & $162.601(8)$ & $34.01(3)$ & $19.32(2)$ & $16.70(2)$ & 10979(9) & 5.28 \\
\hline $0.213(6)$ & $162.586(7)$ & $34.06(3)$ & $19.32(2)$ & $16.69(2)$ & $10978(9)$ & 5.35 \\
\hline $0.216(6)$ & $162.58(1)$ & $34.02(3)$ & $19.32(2)$ & $16.69(2)$ & $10967(9)$ & 5.33 \\
\hline $0.221(6)$ & $162.57(1)$ & $34.03(3)$ & $19.32(2)$ & $16.69(2)$ & 10972(9) & 5.34 \\
\hline $0.223(6)$ & $162.57(1)$ & $33.99(3)$ & $19.33(2)$ & $16.69(2)$ & 10963(9) & 5.44 \\
\hline $0.227(7)$ & $162.56(1)$ & $34.02(3)$ & $19.32(2)$ & $16.67(1)$ & $10956(9)$ & 5.13 \\
\hline $0.243(6)$ & $162.529(9)$ & $33.94(4)$ & $19.34(2)$ & $16.67(2)$ & 10946(9) & 5.20 \\
\hline $0.258(6)$ & $162.498(9)$ & $33.97(3)$ & $19.34(2)$ & $16.64(1)$ & $10938(9)$ & 5.23 \\
\hline
\end{tabular}




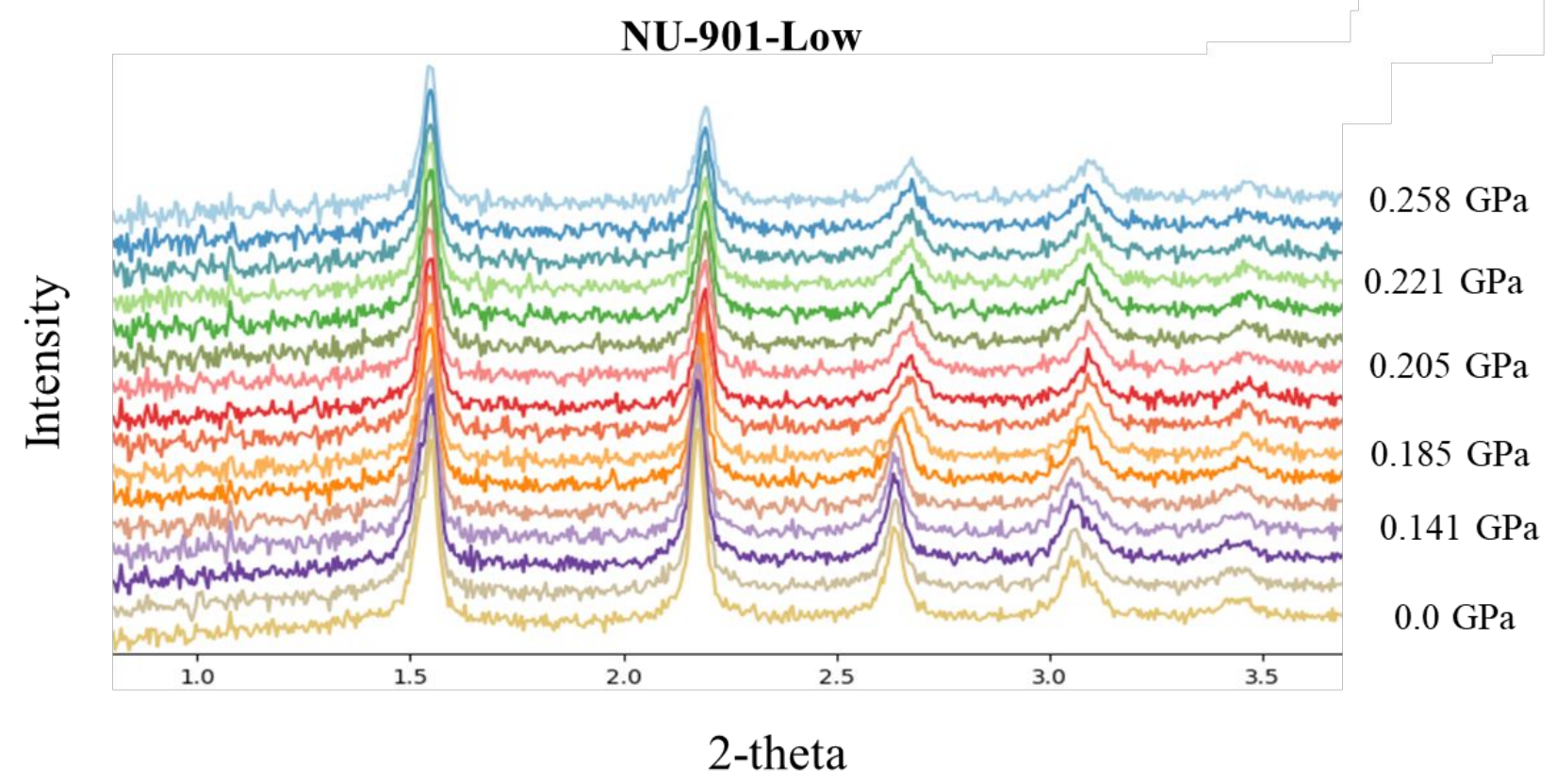

Figure S8. Powder diffraction data for NU-901-L as pressure increases from 0.0-0.258 GPa.

Table S4. Parameters of Le Bail fits to variable pressure powder diffraction data for NU-901-NDCMedium

\begin{tabular}{|c|c|c|c|c|c|c|}
\hline Pressure (Gpa) & $\mathrm{V} \mathrm{CaF}{ }_{2}\left(\AA^{3}\right)$ & a NU-901 $\left(\AA^{3}\right)$ & b NU-901 $\left(\AA^{3}\right)$ & c NU-901 $\left(\AA^{3}\right)$ & V NU-901 $\left(\AA^{3}\right)$ & $\operatorname{Rwp}(\%)$ \\
\hline 0.000 & $163.16(1)$ & $34.49(4)$ & $19.37(3)$ & $16.82(3)$ & $11235(16)$ & 5.48 \\
\hline $0.007(8)$ & $163.14(1)$ & $34.39(4)$ & $19.33(3)$ & $16.87(3)$ & $11214(17)$ & 5.93 \\
\hline $0.030(8)$ & $163.09(1)$ & $34.48(4)$ & $19.28(3)$ & $16.84(3)$ & $11196(16)$ & 5.66 \\
\hline $0.049(8)$ & $163.05(1)$ & $34.41(4)$ & $19.28(3)$ & $16.86(3)$ & $11186(16)$ & 5.89 \\
\hline $0.113(8)$ & $162.93(1)$ & $34.26(4)$ & $19.28(3)$ & $16.83(3)$ & $11117(15)$ & 5.73 \\
\hline $0.164(8)$ & $162.83(1)$ & $34.06(5)$ & $19.28(3)$ & $16.84(3)$ & $11063(15)$ & 6.32 \\
\hline $0.243(7)$ & $162.680(9)$ & $34.01(5)$ & $19.29(3)$ & $16.77(3)$ & $11003(16)$ & 6.73 \\
\hline $0.380(9)$ & $162.41(1)$ & $33.82(6)$ & $19.18(2)$ & $16.84(3)$ & $10922(16)$ & 7.03 \\
\hline $0.486(8)$ & $162.21(1)$ & $33.90(6)$ & $19.13(3)$ & $16.75(3)$ & 10864(18) & 6.96 \\
\hline $0.664(8)$ & $161.87(1)$ & $33.81(6)$ & $19.04(2)$ & $16.70(3)$ & 10753(19) & 7.23 \\
\hline $0.847(8)$ & $161.52(1)$ & $33.60(5)$ & $18.74(1)$ & $16.78(3)$ & $10573(17)$ & 7.22 \\
\hline $1.053(8)$ & $161.13(1)$ & $33.27(5)$ & $18.76(2)$ & $16.73(3)$ & $10436(18)$ & 7.72 \\
\hline
\end{tabular}




\section{NU-901-Medium}

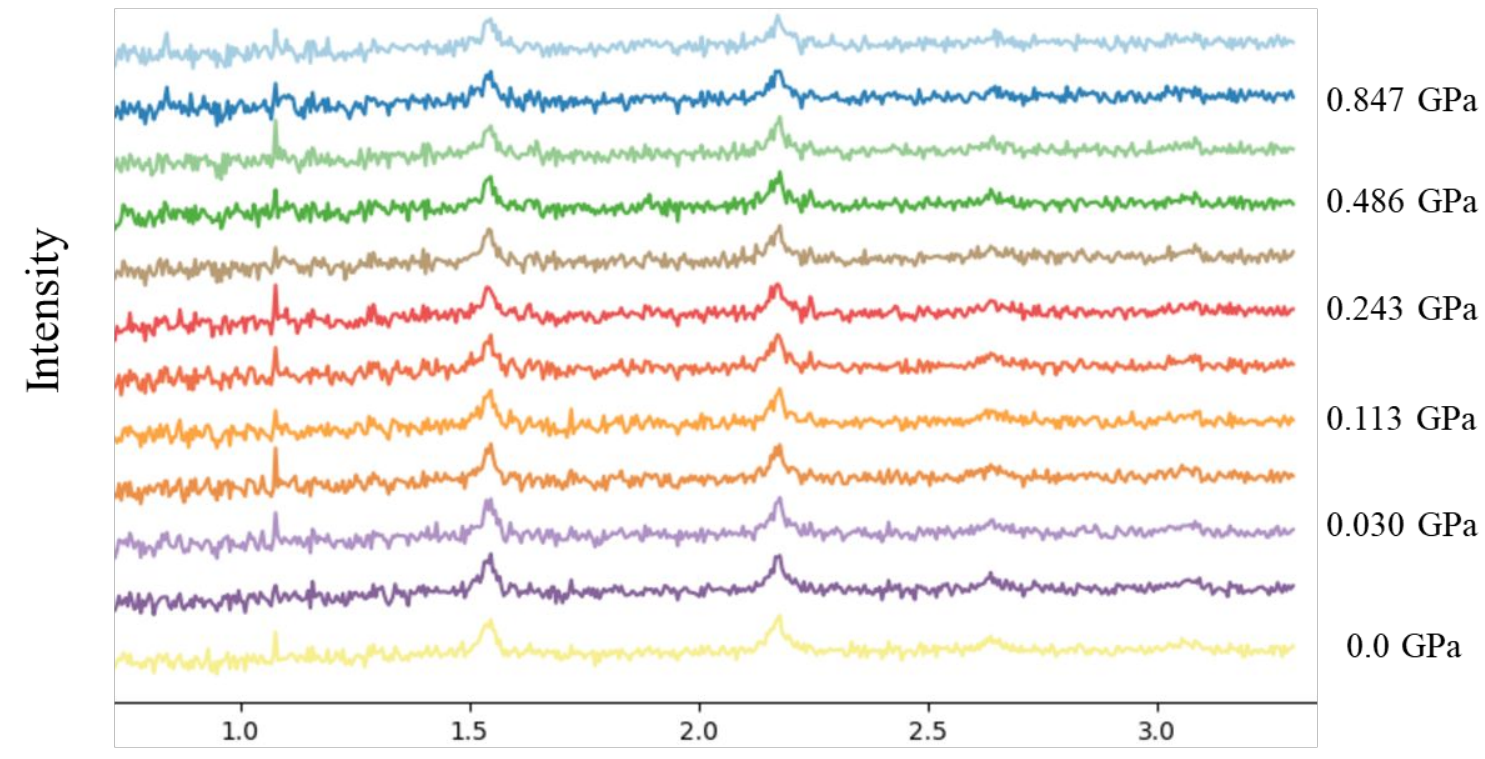

2-theta

Figure S9. Powder diffraction data for NU-901-M as pressure increases from 0.0-1.053 GPa. 
Table S5. Parameters of Le Bail fits to variable pressure powder diffraction data for NU-901-NDCHigh

\begin{tabular}{|c|c|c|c|c|c|c|}
\hline Pressure (Gpa) & $\mathrm{VCaF}_{2}\left(\AA^{3}\right)$ & a NU-901 $\left(\AA^{3}\right)$ & b NU-901 $\left(\AA^{3}\right)$ & c NU-901 $\left(\AA^{3}\right)$ & V NU-901 $\left(\AA^{3}\right)$ & Rwp (\%) \\
\hline 0.000 & $163.094(8)$ & $34.24(2)$ & $19.37(2)$ & $16.93(2)$ & $11229(6)$ & 5.48 \\
\hline $0.002(6)$ & $163.082(8)$ & $34.22(2)$ & $19.38(2)$ & $16.93(1)$ & $11226(7)$ & 5.78 \\
\hline $0.006(6)$ & $163.075(8)$ & $34.23(2)$ & $19.39(2)$ & $16.91(1)$ & $11222(7)$ & 5.61 \\
\hline $0.027(6)$ & $163.034(8)$ & $34.18(2)$ & $19.37(2)$ & $16.92(1)$ & $11204(6)$ & 5.54 \\
\hline $0.047(5)$ & $162.995(7)$ & $34.15(2)$ & $19.37(2)$ & $16.92(1)$ & $11194(6)$ & 5.46 \\
\hline $0.053(6)$ & $162.984(8)$ & $34.14(2)$ & $19.38(2)$ & $16.90(1)$ & $11184(6)$ & 5.45 \\
\hline $0.058(6)$ & 162.973(9) & $34.13(2)$ & $19.39(2)$ & $16.90(1)$ & $11182(7)$ & 5.52 \\
\hline $0.073(6)$ & $162.946(9)$ & $34.12(2)$ & $19.39(2)$ & $16.89(1)$ & $11175(6)$ & 5.48 \\
\hline $0.087(6)$ & 162.917(7) & $34.13(2)$ & $19.37(2)$ & $16.89(1)$ & $11165(7)$ & 5.70 \\
\hline $0.101(5)$ & $162.890(6)$ & $34.10(2)$ & $19.36(2)$ & $16.88(1)$ & $11149(7)$ & 5.70 \\
\hline $0.102(5)$ & $162.889(6)$ & $34.11(2)$ & $19.37(2)$ & $16.88(1)$ & $11158(7)$ & 5.60 \\
\hline $0.106(6)$ & $162.881(7)$ & $34.10(2)$ & $19.38(2)$ & $16.87(1)$ & $11150(7)$ & 5.93 \\
\hline $0.116(6)$ & 162.861(7) & $34.09(2)$ & $19.37(2)$ & $16.87(1)$ & $11141(7)$ & 5.56 \\
\hline $0.130(5)$ & $162.835(7)$ & $34.08(2)$ & $19.37(2)$ & $16.87(1)$ & $11135(7)$ & 5.54 \\
\hline $0.142(5)$ & $162.812(7)$ & $34.07(2)$ & $19.36(2)$ & $16.86(1)$ & $11124(7)$ & 5.76 \\
\hline $0.148(5)$ & $162.799(7)$ & $34.06(2)$ & $19.37(2)$ & $16.85(1)$ & $11120(7)$ & 5.94 \\
\hline $0.154(5)$ & $162.788(7)$ & $34.07(2)$ & $19.37(2)$ & $16.85(1)$ & $11118(7)$ & 5.66 \\
\hline $0.156(5)$ & $162.785(7)$ & $34.03(2)$ & $19.37(2)$ & $16.86(1)$ & 11111(7) & 5.88 \\
\hline $0.168(5)$ & $162.762(7)$ & $34.05(2)$ & $19.36(2)$ & $16.85(1)$ & $11108(7)$ & 5.63 \\
\hline $0.169(5)$ & $162.759(7)$ & $34.05(2)$ & $19.36(2)$ & $16.84(1)$ & $11102(7)$ & 5.67 \\
\hline $0.183(5)$ & $162.731(7)$ & $34.04(2)$ & $19.35(2)$ & $16.84(1)$ & $11093(7)$ & 5.58 \\
\hline $0.181(5)$ & $162.730(7)$ & $34.02(2)$ & $19.35(2)$ & $16.83(1)$ & $11082(7)$ & 5.46 \\
\hline $0.200(5)$ & $162.699(7)$ & $34.01(2)$ & $19.35(2)$ & $16.83(1)$ & $11075(7)$ & 5.61 \\
\hline $0.210(5)$ & $162.680(7)$ & $34.02(2)$ & $19.34(2)$ & $16.82(1)$ & $11068(8)$ & 5.97 \\
\hline $0.233(6)$ & $162.635(8)$ & $34.00(2)$ & $19.35(2)$ & $16.80(1)$ & $11055(8)$ & 5.99 \\
\hline $0.246(6)$ & $162.610(7)$ & $33.96(2)$ & $19.33(2)$ & $16.82(1)$ & $11041(8)$ & 5.85 \\
\hline
\end{tabular}




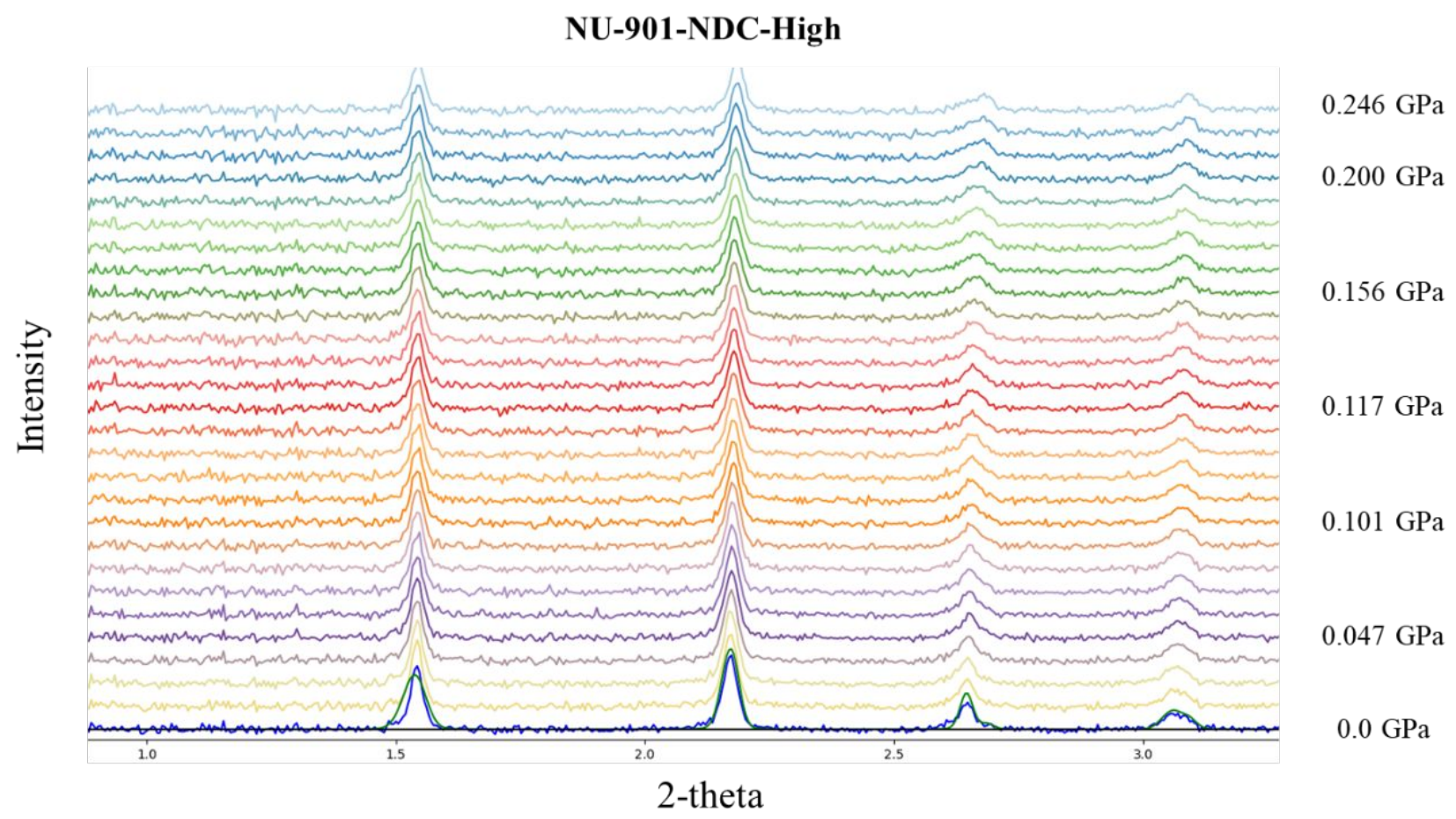

Figure S10. Powder diffraction data for NU-901-H as pressure increases from 0.0-0.246 GPa.

Table S6. Parameters of Le Bail fits to variable pressure powder diffraction data for NU-901-NDCLow (Sample Repeated)

\begin{tabular}{|c|c|c|c|c|c|c|}
\hline Pressure (Gpa) & $\mathrm{VCaF}\left(\mathrm{A}_{2}\left(\AA^{3}\right)\right.$ & a NU-901 $\left(\AA^{3}\right)$ & b NU-901 $\left(\AA^{3}\right)$ & c NU-901 $\left(\AA^{3}\right)$ & V NU-901 $\left(\AA^{3}\right)$ & Rwp (\%) \\
\hline $0.00(3)$ & $163.250(4)$ & $34.46(2)$ & $19.38(1)$ & $16.76(1)$ & 11193(8) & 4.84 \\
\hline $0.009(3)$ & $163.225(5)$ & $34.42(2)$ & 19.37(1) & $16.76(1)$ & $11180(8)$ & 4.75 \\
\hline $0.039(3)$ & $163.168(5)$ & $34.38(2)$ & $19.38(1)$ & $16.76(1)$ & $11164(8)$ & 4.76 \\
\hline $0.055(3)$ & $163.136(5)$ & $34.37(2)$ & $19.37(2)$ & $16.76(1)$ & $11155(8)$ & 4.95 \\
\hline $0.066(3)$ & $163.115(5)$ & $34.36(2)$ & 19.37(1) & $16.75(1)$ & $11147(8)$ & 4.74 \\
\hline $0.084(3)$ & $163.079(5)$ & $34.32(2)$ & $19.34(2)$ & $16.75(1)$ & $11121(8)$ & 4.53 \\
\hline $0.118(4)$ & $163.013(6)$ & $34.28(2)$ & $19.34(2)$ & $16.75(1)$ & $11100(8)$ & 4.46 \\
\hline $0.165(4)$ & $162.924(6)$ & $34.18(2)$ & $19.35(1)$ & $16.72(1)$ & $11056(7)$ & 4.31 \\
\hline $0.204(3)$ & $162.847(5)$ & $34.10(2)$ & $19.32(2)$ & $16.71(1)$ & $11009(7)$ & 4.40 \\
\hline $0.257(3)$ & $162.745(4)$ & $34.07(2)$ & 19.29(1) & $16.68(1)$ & 10961(8) & 4.45 \\
\hline $0.332(3)$ & $162.600(5)$ & $34.07(2)$ & $19.27(1)$ & $16.63(1)$ & $10916(8)$ & 4.25 \\
\hline $0.432(3)$ & $162.409(5)$ & $34.07(2)$ & $19.24(1)$ & $16.56(1)$ & 10859(9) & 4.22 \\
\hline $0.504(3)$ & $162.270(5)$ & $34.08(2)$ & $19.23(1)$ & $16.50(1)$ & $10813(9)$ & 4.26 \\
\hline
\end{tabular}




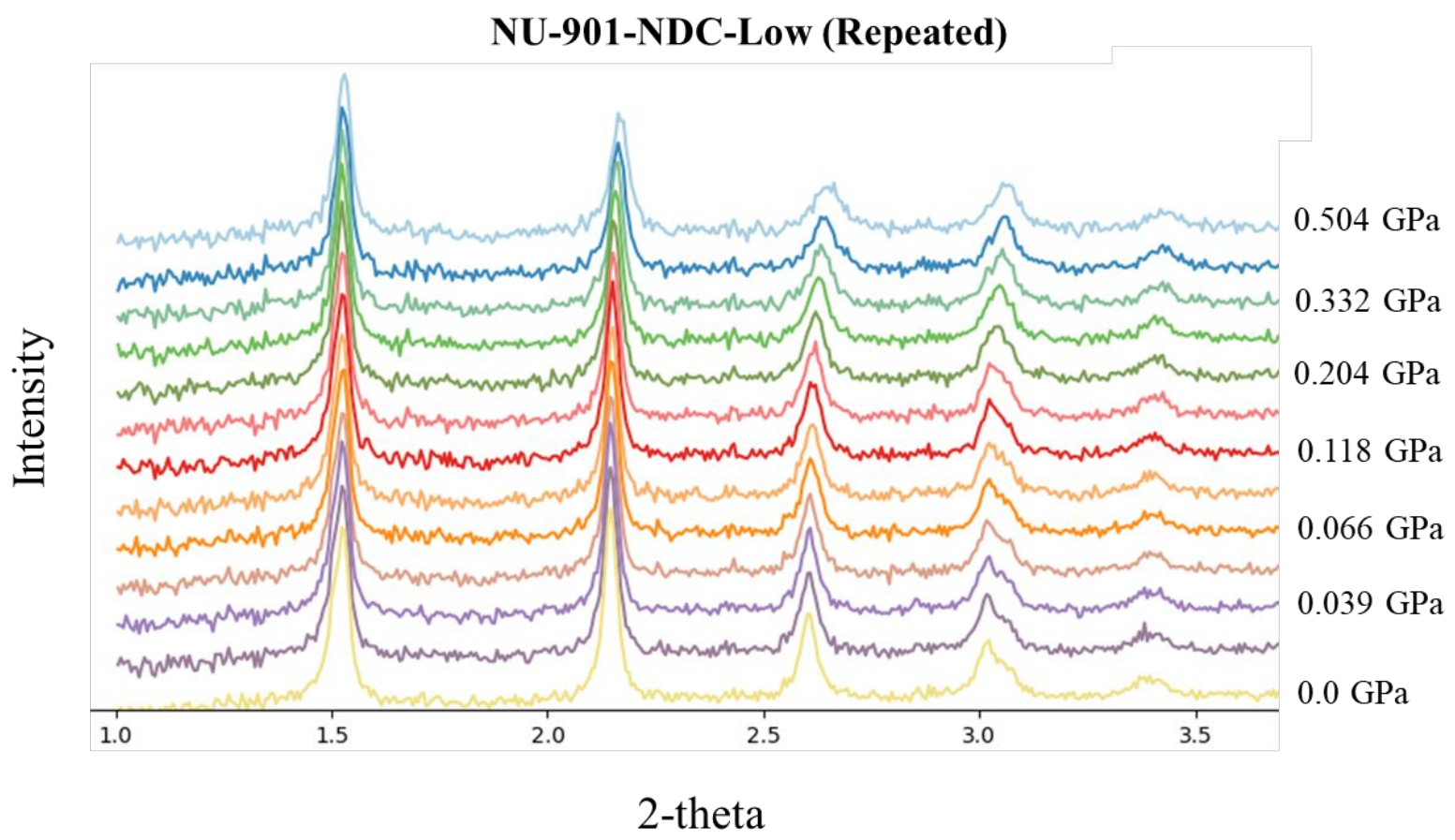

Figure S11. Powder diffraction data for NU-901-L (Sample Repeated) as pressure increases from $0.0-0.504 \mathrm{GPa}$.

Table S7. Parameters of Le Bail fits to variable pressure powder diffraction data for NU-901-NDCMedium (Sample Repeated)

\begin{tabular}{|ccccccc|}
\hline Pressure (Gpa) & $\mathbf{V} \mathbf{C a F}_{\mathbf{2}}\left(\AA^{\mathbf{3}}\right)$ & $\mathbf{a} \mathbf{N U - 9 0 1}\left(\mathbf{\AA}^{\mathbf{3}}\right)$ & $\mathbf{b} \mathbf{~ N U - 9 0 1}\left(\AA^{\mathbf{3}}\right)$ & $\mathbf{c} \mathbf{~ N U - 9 0 1}\left(\AA^{\mathbf{3}}\right)$ & $\mathbf{V} \mathbf{N U - 9 0 1}\left(\AA^{\mathbf{3}}\right)$ & $\mathbf{R w p}(\%)$ \\
\hline $0.00(1)$ & $163.18(2)$ & $34.19(1)$ & $19.29(1)$ & $16.906(9)$ & $11152(5)$ & 4.45 \\
$0.043(9)$ & $163.089(4)$ & $34.08(1)$ & $19.30(1)$ & $16.899(9)$ & $11117(5)$ & 4.26 \\
$0.097(9)$ & $162.986(4)$ & $33.98(2)$ & $19.32(1)$ & $16.86(1)$ & $11069(5)$ & 4.48 \\
$0.197(9)$ & $162.792(4)$ & $33.90(2)$ & $19.28(1)$ & $16.81(1)$ & $10987(5)$ & 4.46 \\
$0.315(9)$ & $162.564(4)$ & $33.88(2)$ & $19.17(1)$ & $16.78(1)$ & $10901(7)$ & 4.60 \\
$0.373(9)$ & $162.452(4)$ & $33.87(2)$ & $19.10(1)$ & $16.78(1)$ & $10853(7)$ & 4.62 \\
$0.459(9)$ & $162.288(4)$ & $33.85(2)$ & $19.04(1)$ & $16.77(1)$ & $10805(8)$ & 4.84 \\
$0.547(9)$ & $162.119(4)$ & $33.82(2)$ & $18.95(1)$ & $16.76(1)$ & $10740(8)$ & 4.80 \\
$0.624(9)$ & $161.971(5)$ & $33.79(2)$ & $18.87(1)$ & $16.74(1)$ & $10674(9)$ & 4.82 \\
\hline
\end{tabular}




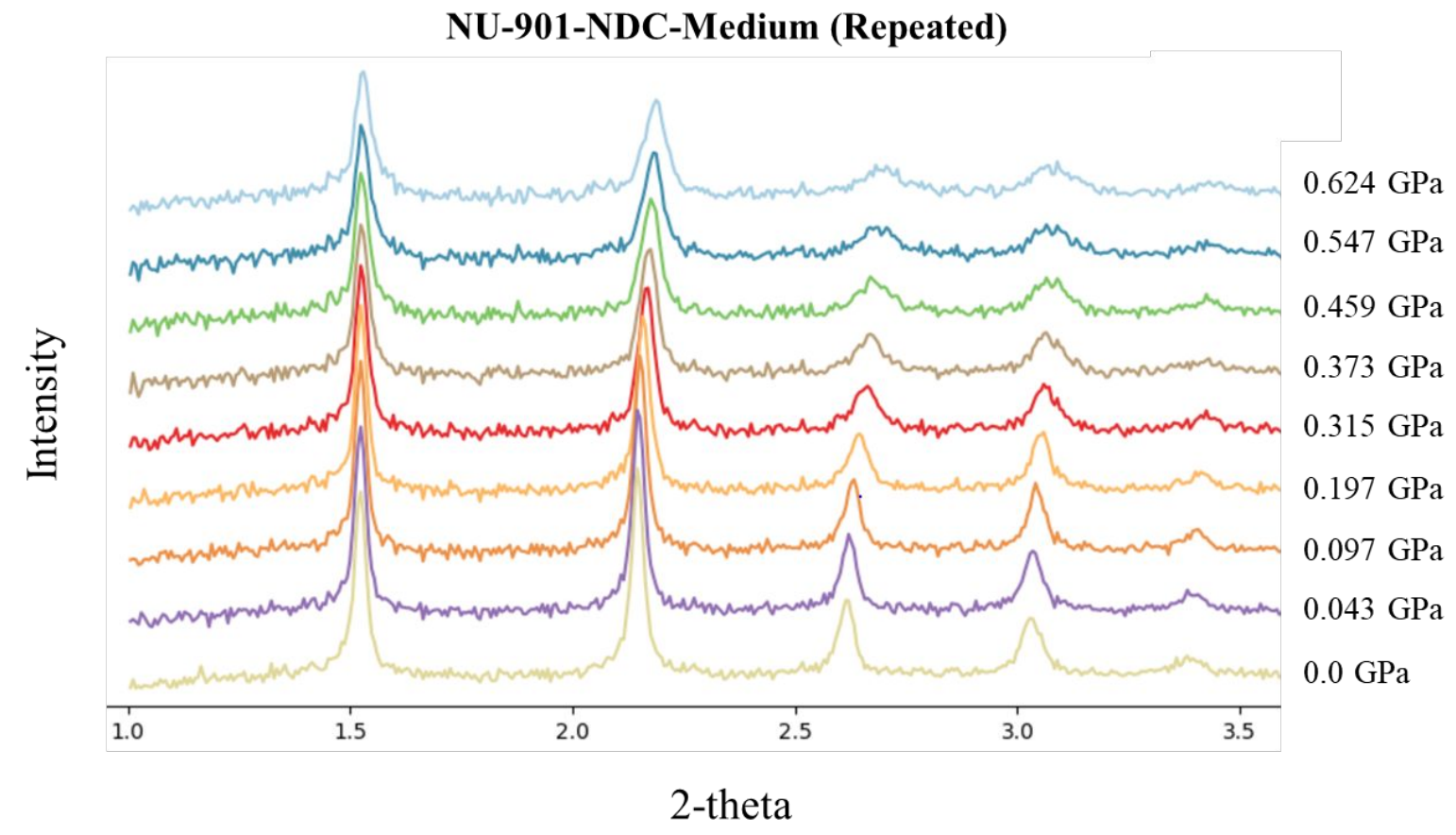

Figure S12. Powder diffraction data for NU-901-M (Repeated) as pressure increases from 0.0$0.624 \mathrm{GPa}$. 


\section{References}

1. Wang, T. C.; Vermeulen, N. A.; Kim, I. S.; Martinson, A. B. F.; Stoddart, J. F.; Hupp, J. T.; Farha, O. K., Scalable Synthesis and Post-Modification of a Mesoporous Metal-Organic Framework Called NU-1000. Nat. Protoc. 2016, 11 (1), 149-162.

2. Garibay, S. J.; Iordanov, I.; Islamoglu, T.; DeCoste, J. B.; Farha, O. K., Synthesis and Functionalization of Phase-Pure NU-901 for Enhanced $\mathrm{CO}_{2}$ Adsorption: The Influence of a Zirconium Salt and Modulator on the Topology and Phase Purity. CrystEngComm 2018, 20 (44), 7066-7070.

3. Yakovenko, A. A.; Reibenspies, J. H.; Bhuvanesh, N.; Zhou, H.-C., Generation and Applications of Structure Envelopes for Porous Metal-Organic Frameworks. J. Appl. Crystallogr. 2013, 46 (2), 346-353.

4. Yakovenko, A. A.; Wei, Z.; Wriedt, M.; Li, J.-R.; Halder, G. J.; Zhou, H.-C., Study of Guest Molecules in Metal-Organic Frameworks by Powder X-Ray Diffraction: Analysis of Difference Envelope Density. Cryst. Growth Des 2014, 14 (11), 5397-5407.

5. Hrubiak, R.; Sinogeikin, S.; Rod, E.; Shen, G., The Laser Micro-Machining System for Diamond Anvil Cell Experiments and General Precision Machining Applications at the High Pressure Collaborative Access Team. Rev. Sci. Instrum. 2015, 86 (7), 072202.

6. Hammersley, A. P.; Svensson, S. O.; Hanfland, M.; Fitch, A. N.; Hausermann, D., TwoDimensional Detector Software: From Real Detector to Idealised Image or Two-Theta Scan. High Press Res. 1996, 14 (4-6), 235-248.

7. Toby, B. H.; Von Dreele, R. B., Gsas-Ii: The Genesis of a Modern Open-Source All Purpose Crystallography Software Package. J. Appl. Crystallogr. 2013, 46 (2), 544-549.

8. Angel, R.; Gonzalez-Platas, J.; Alvaro, M., EoSFit7c and a Fortran Module (Library) for Equation of State Calculations. Z. Kristallogr. Cryst. Mater. 2014, 229 (5): 405-419.

9. Gonzalez-Platas, J.; Alvaro, M.; Nestola, F.; Angel, R., Eosfit7-Gui: A New Graphical User Interface for Equation of State Calculations, Analyses and Teaching. J. Appl. Crystallogr. 2016, 49 (4), 1377-1382. 